\title{
MYOMA AND PREGNANCY.
}

By SIR W. J. SMYLY, M.D., F.R.C.P.I.,

Ex-Master of the Rotunda Hospital.

[Read in the Section of Obstetrics, November 13, 1908.]

MY object in this communication is to record a case in which child-bearing was complicated by a myoma of the uterus; but I shall first give a short account of the subject generally.

We are all familiar with myomata in pregnant women who are approaching the end of the child-bearing period, which, as a rule, cause little or no trouble and are soon forgotten. The few, however, which do cause trouble are noted and published, sor that to collect and tabulate the cases which appear from time to time in medical journals would give an altogether erroneous impression of the seriousness of this complication. On the other hand, it must not be forgotten that occasionally serious complications do occur in these cases which tax the skill and resourcefulness of the attendant to the utmost.

We may consider the subject from two aspects. First, the effect of pregnancy on the tumours, and secondly the effect of the tumours upon the course of pregnancy, childbirth, and the puerperium.

1. The occurrence of pregnancy in a myomatous uterus causes a change in the position, size, shape, and consistence of the tumours. As the uterus enlarges and rises out of the pelvis so do the tumours attached to its body. Even those which are attached to the lower segment are drawn upwards towards the end of pregnancy, or even during labour, and only cervical tumours, or those which 
grow into the cellular tissue at the sides of the uterus, maintain their position or are driven downwards during. labour, and are, therefore, the most likely to cause trouble. As the uterus grows larger and softer so do the tumours attached to it. This change in size and consistence is due to development of their tissues or œdema, the latter condition sometimes causing a rapid enlargement out of proportion to the enlargement of the uterus. At the same time they lose their globular shape, becoming flattened out as the uterus expands, and are more difficult to differentiate from the normal tissues. Necrotic changes are rare during pregnancy, and are generally caused by twisting of the pedicle, but are more common during the puerperium, and may then result in sloughing, suppuration and general sepsis. In the puerperium as the uterus involutes the tumours return to their original condition, and in a few cases have completely disappeared.

2. If we now turn to the effects of myomatous tumours. upon the course of pregnancy, childbirth and the puerperium one will find that during pregnancy they rarely give rise to serious complications, but that cases have been recorded in considerable numbers in which they caused hæmorrhages, abortions, placenta prævia, ectopic pregnancy, intestinal obstruction, ischuria and obstruction of the ureters, with consequent hydronephrosis, twisting of the pedicle with peritonitis, and serious pressure symptoms from the great size of the growth, or from retroversion and impaction of the uterus in the pelvis.

A fact of considerable importance in connection with. pregnancy complicated by myomata is that not infrequentiy there is great difficulty in forming a correct diagnosis: and, as a matter of fact, a large number of uter have actually been removed in total ignorance of 
the complicating pregnancy. These mistakes are partly due to the fact that many of the symptoms are common to both conditions, such as enlargement of the uterus, blueing of the vagina, and the uterine souffle.

Hæmorrhage is a common occurrence in pregnancy complicated by myomata, especially in the early months, and all operators should lay to heart the warning given by Olhaussen " that a rapid enlargement of a myomatous uterus accompanied by hæmorrhage should always excite a suspicion of pregnancy." In these early cases, too, when the uterus is completely imbedded in tumours, the peculiar softening so suggestive of its condition may be difficult, or even impossible, to detect.

During labour, tumours of the body of the uterus seldom give rise to trouble, and are often overlooked or mistaken for fœetal parts, from which, howerer, with a little care, they can be easily distinguished, because they never change their position, and cannot be pressed back into the uterine cavity. Not infrequently, however, they cause inertia, and when situated in the lower segment or cervix the latter dilates with difficulty, so that labour may be prolonged over several days. As a rule, nature overcomes the difficulty by drawing up the tumour, or it is pushed down or flattened out by the presenting part. But large tumours adherent in the pouch of Douglas or wbich have developed into the subserous areolar tissue, may cause serious, and even insuperable, obstacles to delivery. Malpresentations are frequent in these cases, and are due either to deformity of the uterine cavity or to the presenting part being unable to enter the pelvic brim.

Placenta prævia occurs with such relative frequency as almost to prove the existence of cause and effect; and the most probable explanation is that the mucous membrane 
covering the tumour affords such an unfavourable surface that the formation of the placenta is not limited to the serotina, but involves a much wider area, including the reflexa; and, therefore, more likely to extend into the lower segment.

In the third stage myomata cause trouble much more frequently than in either of the other stages of labour. Imperfect contraction and retraction of the muscular tissue, or retention in whole or in part of the placenta, are frequent causes of hæmorrhage.

Adhesion of the placenta when implanted upon the tumour itself is especially serious, because the membrane covering submucous tumours is generally so thin that instead of imbedding itself in the decidua the ovum passes completely through it, and the placenta becomes so intimately connected with the tissue of the tumour that separation may be found difficult or even impossible, and cases of this kind bave been reported in which fatal hæmorrhage has followed removal of the placenta, and others in which extirpation of the uterus has proved the only possible way out of the difficulty. Inversion of the uterus due to fundal attachment of the tumour is a wellknown complication.

More important, because more frequent, than any other complication is hæmorrhage after labour is completed. It may occur at any time during the puerperium, so that it is difficult to say when a patient with a myomatous uterus is safe. Probably the most frequent and peculiar kind of hæmorrhage is that described by Barnes as paulopost-partum hæmorrhage. I recorded a fatal case of this kind in the Rotunda Hospital Reports. The patient was delivered in the evening after a normal labour; at midnight there was a violent hæmorrhage, which was controlled; at six in the morning a second and fatal hæmor- 
rhage occurred. A submucous myoma was found at the post-mortem examination.

Septic complications due to retention of lochia, portions of the placenta or membranes, or to sloughing and suppuration of the tumours themselves are by no means infrequent.

The treatment of these cases, as is evident from what I have already stated, is always a matter of importance, and often of grave anxiety to the attendant.

During pregnancy interference is seldom called for, although tumours have been successfully removed without interrupting gestation, but excepting those rare cases in which extreme pressure symptoms, twisting of the pedicle, or sloughing of the tumour render operative interference necessary it is better to postpone such measures until full term. The induction of abortion, frequently resorted to in former times, has now been altogether abandoned.

At term and during labour those tumours which cause no obstruction or other serious trouble need no special treatment.

Polypi should be removed and cervical myomata enucleated; but tumours which grow into the cellular tissue can seldom be removed per vaginam without great danger.

When the tumour is so large and in such a position as to cause serious obstruction to the passage of the child we have a number of methods of delivery to select from, and the best choice is often very difficult to determine.

We know by experience that in such cases nature unassisted will often succeed in overcoming an obstacle which seemed insuperable, as was well illustrated by a remarkable case reported to us by Dr. Kidd, in which a large tumour appeared to fill the pelvic cavity, and to 
offer an insuperable obstacle to delivery. He performed a successful Cæsarean section. Yet in a subsequent confinement this same woman delivered herself spontaneously. We should, therefore, make it a rule to wait as long as spontaneous delivery is possible, but not so long as to render operative interference hopeless.

Efforts to drag the foetus past the obstruction by forceps, version and extraction, or after perforation, are liable to cause irreparable damage, and are in general to be condemned.

As regards abdominal operations, Cæsarean section alone is seldom sufficient. It is more generally advisable to combine it with enucleation of the tumour or extirpation of the uterus. As a rule panhysterectomy has proved more successful than the supra-vaginal operation, because after a prolonged labour the uterus is often more or less septic, and the retention of a septic stump is not only in itself a source of danger, but its complete removal affords better drainage. Another reason for the complete operation is that the tumours which cause obstruction are always situated so low in the uterus that it is difficult to obtain a stump altogether free from disease. When the fortus is known to be dead it is better to remove the uterus unopened. In some cases where it is evident, after opening the abdomen, that the removal of the tumour alone is possible, it has been recommended first to pull up the tumour out of the way, then to deliver per vias naturales, and lastly to remove the tumour.

After delivery it is scarcely necessary to point out that post-partum hæmorrhage must be looked for and free drainage of discharges secured; and in no other class of cases is complete asepsis from start to finish a more absolute necessity. I shall now proceed to the case :- -

In September, 1903, I was consulted by a lady, who told me 
that she had been married eight years, but had no children. She complained of severe neuralgic pains in her breasts, especially at the intermenstrual periods, when they became swollen and painful. Menstruation was regular, but very profuse, with expulsion of clots, and lasted a full week, generally, but not always accompanied by pain.

The uterus was found to be enlarged, but no tumour was discovered. I diagnosed the case, and treated it as chronic metritis.

A year afterwards, in September, 1904, she became pregnant, and the pregnancy pursued a normal course. Labour commenced on July 5, 1905, the breech presented, and pains were weak and inefficient.

On the evening of the fourth day the membranes ruptured, and I found the child lying obliquely with the breech in the right iliac fossa. I then made my first internal examination, and found the os three-quarters dilated, and a hand presenting. To the left of the cervix I also found, to my surpise, a tumour as large as my fist, and which I could not push out of the pelvic brim. Having procured Dr. Glenn's assistance, the patient was anæsthetised. I introduced my hand into the uterus and brought down a foot without difficulty, but extraction proved unusually tedious, and the child died before it was completed. Ultimately, however, I succeeded in delivering the dead child without serious injury to the maternal soft parts. After the expulsion of the placenta the uterus contracted well, and for some time there was no hæmorrhage, but after a time the organ relaxed and enlarged, and the discharge became more profuse; the uterus was rubbed and pressed, some clots expelled, and ergot administered with good effect; but after a time the hæmorrhage recurred, and I washed out the uterus with hot water. The benefit was only temporary, but at no time could the loss be called a flooding. There was no injury to the cervix, nor was she at any time bad enough to suggest the advisability of plugging, but still her condition was so unsatisfactory that it was between two and three hours from the birth of the child before we thought it safe to leave the house.

On the fourth day after delivery the lochia became fœtid, and there was a rise of temperature, which on the following 
evening rose to $103^{\circ} \mathrm{F}$, and the vagina was washed out with creolin lotion. The following morning she was no better, so I douched the uterus with 1 per cent. formalin solution. After which the temperature sank to normal and remained so for two days. After that it rose again, and on the eighth day it was so high, and her general condition so unsatisfactory, that I asked for a consultation, and Sir Arthur Macan saw her with me in the evening.

Having discussed the advisability of exploring the uterus to see if anything could have been left behind we finally decided to try the intra-uterine douche again. Next day her temperature was normal, and she gave me no further cause for anxiety.

The numerous complications in this confinement were no doubt all due to the presence of the tumour. The tedious labour was duc to the difficulty in expanding the lower uterine segment. The change in the fortal lie was caused partly by the inability of the presenting part to enter the pelvic brim and partly to the unequal. expansion of the lower segment of the uterus, the left side remaining rigid whilst the right, becoming over-distended and thinned, would yield to the pressure of the presenting part, which consequently became displaced in that direction.

The hand presentation was due to tho lie of the foetus, and its death was caused by obstruction to its delivery and pressure on the cord.

Post-partum hæmorrhage is, as I have already pointed. out, a common occurrence in myomatous uteri, but is not quite so easy to understand in this case where the body of the uterus was healthy, and I am inclined to think that it was due to the tumour acting as a ball valve closing the os and obstructing the escape of discharge, which consequently accumulated in, and, by distending the uterus, acted in antagonism to its retraction. But whether this was the cause of the hæmorrhage or not it certainly must 
have been the cause of the retention and putrefaction of the lochia and the septic fever which accompanied it.

I had, therefore, little difficulty in deciding what advice I should give when, some months later, this lady consulted me again. I explained to her that the tumour, which had then shrunk to about the size of a billiard ball, was at that time doing her no harm, and that unless she again became pregnant would probably cause her no inconvenience; but that in future pregnancies, unless it had been previously removed, she would probably encounter similar dangers to those which she had already passed through.

After some consideration she decided to submit to operation, and accordingly, on Octobex 28, 1905, I opened her abdomen and found the tumour within the left broad ligament, which I incised on its anterior aspect and enucleated the growth from the cellular tissue and uterine wall, into which it was embedded by about onethird of its bulk. Having closed the cavity with fine silk sutures I closed the abdomen, and she made a good recovery.

In 1906 she again became pregnant, went to full term, and on April 1\%, 190\%, delivered herself of a fullydeveloped living child. Her labour was normal, and, excepting a mammary abscess, her convalescence was quite uneventful.

After the operation I shored the tumour to the members of this section as a recent specimen, but I think the very satisfactory ultimate result of the case is sufficent reason for bringing the subject forward again.

Dr. FtrzGibion cited a case in which a fibroid occupied the entire fundus. It flattened out over the foetus, until in the later months it was difficult to differentiate the tumour from the uterine 
contents. The case was watched, and allowed to go to full term, and the woman was delivered of a living child without difficulty.

Dr. Purefor said the difficulty of diagnosticating pregnancy in the first few months in fibroid uterus was a matter of great importance, and should be constantly present to their minds when dealing with such cases. Sir William Smyly's paper illustrated the fact that a woman having a fibroid uterus often passed through several years of married life before pregnancy occurred. In some cases the tumour remained stationary in pregnancy. In some it diminished, as in a case where several small fibroids were palpated at the first pregnancy, while in a second and third pregnancy their presence was difficult to recognise. On the other hand, in some cases the tumour grew very rapidly. His own observations bore out Sir William Smyly's statement that in some cases they removed a fibroid uterus without suspecting it to be gravid. The presence of one or more fibroids would give them trouble chiefly in the third stage. Some observers in England advised patients to avoid matrimony, as pregnancy was fraught with great risk under the circumstances, but that was not their experience in this country.

Dr. Houmes recalled a case in which the patient, after delivery, developed puerperal ulcer, and died. The uterus was studded all over with myoma, and practically every fibroid was either a little abscess sac or had burst.

DR. Rowletre said there was a peculiar form of degeneration in fibroids which occurred frequently, but did not appear to occur apart from pregnancy. In some cases the whole fibroid underwent a marked red pigmentation. In other cases there was much less pink coloration.

Dr. Henry Jelletrt said he had watched a case during the whole of pregnancy a year ago. The patient came about the second month. The uterus was large, soft and flabby on top, and below was hard and irregular. Ho came to the conclusion that there were myomata, and that she was probably pregnant. The myomata were situated apparently as close to the cervix as they could get. Pregnancy went on without trouble, and the myomata preserved their position. After the ninth lunar month the myomata were still close to the cervix, but with a little space between. $\mathrm{He}_{e}$ still thought they would probably offer an 
obstruction to delivery. A fortnight afterwards, however, there were no myomata to be felt, as they had gone up above the symphysis, drawn up during the formation of the lower uterine segment. She had a normal confinement. In another case he operated two years after pregnancy, and found two ordinary intra-mural myomata which had apparently completely died. There was no circulation in them, and they had a complete capsular wall of calcareous concretions.

Dr. AsHe asked what was the position of the placenta in relation to the tumour.

Tr. E. H. TweEd said so far as he knew this case was the first reported in Dublin of myomectomy with subsequent pregnancy. Myomectomy at full term without the removal of the uterus seemed to him an impossible operation in the majority of cases. The hæmorrhage that followed the enucleation of a myoma in a pregnant uterus would be greater than he would like to face. In young married women they would all operate if they were sure they could remove the tumours by myomectomy, but that was. what they were not sure of. He therefore always let a young married woman keep the myoma in the hope that she would become pregnant. He thought their resources were quite adequate to deal with the myomatous condition no matter where situate. It was not always easy to diagnosticate pregnancy when it occurred in a myomatous uterus.

Sir William Smyly, in reply, said the tumour was attached. to the lower uterine segment, and was about the size of a billiard ball. When he opened the broad ligament from the front he had no difficulty in enucleating it. There was considerable hæmorrhage, but he was fortunate in that the first suture included the uterine artery, and he had little trouble afterwards. He did not ligate the ovarian artery separately. He sutured up the rest of tho cavity with silk sutures. 